Monatsschr Kinderheilkd 2003 · [Suppl 1] 151:S4 DOI 10.1007/s00112-003-0786-y

(C) Springer-Verlag 2003

F.H.Sennhauser

\title{
Kongressbericht Dreiländersymposium in Bad Ragaz
}

$\mathbf{G}_{\text {esundheit und Krankheit sind als la- }}$ biles Gleichgewicht die Resultante eines dynamischen Wechselspiels zwischen genetischer Anlage und Umweltfaktoren. Reifungs- und Alterungsprozesse haben zusätzlich eine große modulierende Bedeutung und definieren den entwicklungsabhängigen Zeitpunkt, wann und wie exogene Einflüsse eine Fehlentwicklung fördern oder verhindern. Dieses wachsende Verständnis der meist mehrstufigen pathogenetischen Mechanismen ermöglicht somit eine prozessorientierte und prioritäre Nutzung derartiger Erkenntnisse für früh- und rechtzeitige Prävention im Sinne einer Salutogenese mit dem zunehmend realistischer werdenden Ziel von Gesundheitserhaltung trotz genetisch gegebener Disposition für Gesundheitsstörung oder Krankheit. Die innovative zukunftsorientierte Pädiatrie erfüllt damit einen dualen Auftrag: Sie behandelt Krankheiten und betreut Kranke, gleichermaßen erhält sie aber Gesundheit und berät Gesunde.

Die intrauterine und frühkindliche Ernährung ist ein universell wirksamer und daher besonders bedeutsamer ubiquitärer exogener Einflussfaktor, der im Rahmen der „Fetal-origin-Hypothese“ die Aktualität und das wissenschaftliche Interesse an frühen Ernährungseinflüssen akzentuiert hat: Veränderungen von Ernährung und endokrinen Faktoren während der Fetalphase beeinflussen die weitere und spätere Entwicklung und führen zu permanenten Alterationen von Struktur, Physiologie und Stoffwechsel mit konsekutiver in- dividueller Prädisposition für kardiovaskuläre, metabolische und hormonelle Krankheiten z. T. erst im Erwachsenenalter. Im Rahmen dieses sog., programming“" kann also ein Stimulus oder eine Schädigung während einer sensitiven oder kritischen Entwicklungsphase zu permanenten Langzeitdefekten führen.

Das 8. Dreiländersymposium widmete sich im März 2003 in diesem Kontext der spannenden Frage, welche frühen Ernährungseinflüsse die spätere Immunkompetenz oder Allergieentstehung, das menschliche Wachstum und die Adipositas mit ihrer global zunehmenden Inzidenz und Prävalenz prädestinieren und modulieren - wichtige Fragen mit sowohl medizinischwissenschaftlicher als auch sozioökonomischer Bedeutung.

24.05.2003

F.H. Sennhauser 